\title{
Vorbemerkung zu den beiden folgenden Beiträgen
}

Bereits der erste Internationale Kongreß der Versicherungsmathematiker, der 1895 in Brüssel stattfand, befaßte sich mit der Festlegung einer international verbindlichen Bezeichnungsweise in der Versicherungsmathematik. Damals wurde beschlossen zu prüfen, ob man von der Bezeichnungsweise des Londoner Institute of Actuaries ausgehen und lediglich gewisse Erweiterungen oder Modifikationen vornehmen solle. Auf diese Weise konnte sich bereits der 2. IKVM 1898 in London dieser Frage abschließend widmen. Es wurde eine Bezeichnungsweise vereinbart, wie sie in deutscher Sprache auf S. 633 bis S. 640 der Kongreßberichte des 2. IKVM niedergelegt ist. Die auf dem 14. Kongreß 1954 in Madrid beschlossene und heute geltende Bezeichnungsweise ist nur eine Modifikation dieser versicherungsmathematischen ,Notation" von 1898 (vgl. Blätter Bd. II, S. 367-376).

Ein neues Bild bietet nun der Vorschlag einer europäischen Arbeitsgruppe, wie man den folgenden Seiten entnehmen kann. Er entstand aus den Vorschlägen einer deutschsprachigen Arbeitsgruppe, die dem 18. IKVM, München 1968, vorgelegt wurden. Ihre Uberarbeitung, vor allem der noch nicht kodifizierten Teile der Bezeichnungsweise, führte $\mathrm{zu}$ einer ,,versicherungsmathematischen Veröffentlichungssprache“, die nunmehr dem 19. IKVM, 19. - 24. Juni 1972 in Oslo, vorgelegt werden wird.

Die Grundgedanken und Vorteile der vorgeschlagenen Veröffentlichungssprache erkennt man am schnellsten beim Betrachten der Beispiele auf S. 348 und 349. Eine eingehendere Durchsicht läßt erkennen, daß die Arbeitsgruppe bestrebt war, ein umfassendes Bezeichnungssystem, das eindeutig, in sich widerspruchsfrei und möglichst vollständig ist, aufzubauen. Der darauf folgende Beitrag läßt die Grundkonzeption des Vorschlags am Beispiel der Pensionsversicherung im besonderen deutlich werden.

Schriftleitung 